\title{
A Short-Term Planning Framework for the Operation of Tanker-Based Water Distribution Systems in Urban Areas
}

\author{
Abhilasha Maheshwari ${ }^{\dagger \#}$, Shamik Misra ${ }^{\dagger \#}$, Ravindra D. Gudi ${ }^{\dagger, *}$, \\ Senthilmurugan Subbiah \\ ${ }^{\dagger}$ Department of Chemical Engineering, Indian Institute of Technology Bombay, Powai, Mumbai \\ - 400076, India \\ * Department of Chemical Engineering, Indian Institute of Technology Guwahati, Guwahati, \\ Assam-781039, India
}

\section{Supplementary Information}

\section{Industrial \& Engineering Chemistry Research}

\#Both authors have contributed equally

*To whom correspondence must be addressed: Ravindra D. Gudi (E-mail: ravigudi@iitb.ac.in, Department of Chemical Engineering, IIT Bombay, Mumbai, India, contact: +91-022-2576-7231) 
Following data is assumed to describe the application case study presented in the paper:

Table S1:Water Sources and Treatment Facility Description

\begin{tabular}{|l|l|l|l|l|l|l|}
\hline Source ID & $\begin{array}{l}\text { Source } \\
\text { Type }\end{array}$ & $\begin{array}{l}\text { Treatment Cost } \\
\text { per KL (Rs) }\end{array}$ & $\begin{array}{l}\text { Uptime } \\
\text { of TF (h) }\end{array}$ & $\begin{array}{l}\text { Downtime } \\
\text { of TF (h) }\end{array}$ & $\begin{array}{l}\text { Throughput } \\
\text { of TF (KL/H) }\end{array}$ & $\begin{array}{l}\text { Disinfection } \\
\text { Time (h) }\end{array}$ \\
\hline FW2 & FW & & & & & 1 \\
\hline FW3 & FW & & & & & 1 \\
\hline GW1 & GW & & & & & \\
\hline GW3 & GW & & & & & \\
\hline GW4 & GW & & & & & \\
\hline TF1 & TF & 80 & 10 & 4 & 50 & \\
\hline TF2 & TF & 50 & 10 & 4 & 80 & \\
\hline
\end{tabular}

Table S2:Ground Water Extraction Limitations

\begin{tabular}{|l|l|}
\hline SourcelD & $\begin{array}{l}\text { Max Extraction } \\
\text { Limit (KL/h) }\end{array}$ \\
\hline GW1 & 20 \\
\hline GW3 & 20 \\
\hline GW4 & 25 \\
\hline
\end{tabular}

Table S3: Source-Treatment plant distance

\begin{tabular}{|l|l|l|}
\hline SourcelD & TreatmentfacilityID & Distance $(\mathbf{K m})$ \\
\hline GW1 & TF1 & 55 \\
\hline GW1 & TF2 & 60 \\
\hline GW3 & TF1 & 48 \\
\hline GW3 & TF2 & 35 \\
\hline GW4 & TF1 & 48 \\
\hline GW4 & TF2 & 52 \\
\hline
\end{tabular}

Table S4: Water Product States Description

\begin{tabular}{|l|l|l|}
\hline Product ID & Product Description & Final Product Flag \\
\hline DPW & Domestic Purpose water & 1 \\
\hline UPDW & Ultra-pure drinking water & 1 \\
\hline RW & Untreated ground water & \\
\hline
\end{tabular}


Table S5: Customer Description

\begin{tabular}{|l|l|l|l|}
\hline $\begin{array}{l}\text { Customer } \\
\text { ID }\end{array}$ & Customer Description & Customer Type & $\begin{array}{l}\text { Customer Distribution } \\
\text { Time (h) }\end{array}$ \\
\hline HHC1 & Household Customers in Region 1 & Residential & 4 \\
\hline CC1 & Commercial Customers in Region 1 & Hotels/offices & 3 \\
\hline HC1 & Hospital Customers in Region 1 & Hospitals & 2 \\
\hline HHC2 & Household Customers in Region 2 & Residential & 4 \\
\hline CC2 & Commercial Customers in Region 2 & Hotels/offices & 3 \\
\hline HC2 & Hospital Customers in Region 2 & Hospitals & 2 \\
\hline HHC3 & Household Customers in Region 3 & Residential & 4 \\
\hline CC3 & Commercial Customers in Region 3 & Hotels/offices & 3 \\
\hline HC3 & Hospital Customers in Region 3 & Hospitals & 2 \\
\hline
\end{tabular}

Table S6: Source-Customer Distance

\begin{tabular}{|l|l|l|}
\hline SourcelD & CustomerID & Distance (Km) \\
\hline FW2 & HHC1 & 30 \\
\hline FW2 & CC1 & 40 \\
\hline FW2 & HC1 & 15 \\
\hline FW3 & HHC1 & 20 \\
\hline FW3 & CC1 & 25 \\
\hline FW3 & HC1 & 30 \\
\hline TF1 & HHC1 & 12 \\
\hline TF1 & CC1 & 13 \\
\hline TF1 & HC1 & 10 \\
\hline TF2 & HHC1 & 51 \\
\hline TF2 & CC1 & 36 \\
\hline TF2 & HC1 & 39 \\
\hline FW2 & HHC2 & 38 \\
\hline FW2 & CC2 & 35 \\
\hline FW2 & HC2 & 34 \\
\hline FW3 & HHC2 & 15 \\
\hline FW3 & CC2 & 55 \\
\hline FW3 & HC2 & 20 \\
\hline TF1 & HHC2 & 48 \\
\hline TF1 & CC2 & 44 \\
\hline TF1 & HC2 & 42 \\
\hline TF2 & HHC2 & 15 \\
\hline TF2 & CC2 & 10 \\
\hline TF2 & HC2 & 15 \\
\hline FW2 & HHC3 & 10 \\
\hline & & \\
\hline
\end{tabular}




\begin{tabular}{|l|l|l|}
\hline FW2 & CC3 & 30 \\
\hline FW2 & HC3 & 10 \\
\hline FW3 & HHC3 & 33 \\
\hline FW3 & CC3 & 43 \\
\hline FW3 & HC3 & 25 \\
\hline TF1 & HHC3 & 26 \\
\hline TF1 & CC3 & 28 \\
\hline TF1 & HC3 & 26 \\
\hline TF2 & HHC3 & 35 \\
\hline TF2 & CC3 & 20 \\
\hline TF2 & HC3 & 25 \\
\hline
\end{tabular}

Table S7: Water Tanker Specifications

\begin{tabular}{|l|l|l|l|l|}
\hline TruckID & $\begin{array}{l}\text { Average Speed } \\
(\mathbf{K m} / \mathbf{h r})\end{array}$ & $\begin{array}{l}\text { Capacity } \\
\text { (KL) }\end{array}$ & Cost Per Km (Rs) & $\begin{array}{l}\text { Distribution Cost per } \\
\text { quantity }\end{array}$ \\
\hline $3 T$ & 25 & 3 & 12 & 4 \\
\hline $6 \mathrm{~T}$ & 20 & 6 & 14 & 2.34 \\
\hline $10 \mathrm{~T}$ & 15 & 10 & 23 & 2.3 \\
\hline
\end{tabular}

Table S8: Distribution Tanker Details

\begin{tabular}{|l|l|l|l|}
\hline TruckID & ProductID & TruckType & $\begin{array}{l}\text { Extra Tanker Purchase } \\
\text { Penalty Cost (Rs) }\end{array}$ \\
\hline $3 T$ & DPW & SSD & 10000 \\
\hline $6 T$ & DPW & SSD & 20000 \\
\hline $10 T$ & DPW & SSD & 50000 \\
\hline $3 T$ & UPDW & SSU & 10000 \\
\hline $6 T$ & UPDW & SSU & 20000 \\
\hline $10 T$ & UPDW & SSU & 50000 \\
\hline $6 T$ & RW & ESS & 40000 \\
\hline $10 T$ & RW & ESS & 80000 \\
\hline
\end{tabular}

Table S9: Tanker Availability Details for each region

\begin{tabular}{|l|l|l|l|}
\hline $\begin{array}{l}\text { Region } \\
\text { ID }\end{array}$ & Product ID & $\begin{array}{l}\text { Tanker } \\
\text { ID }\end{array}$ & $\begin{array}{l}\text { Number of Tanker } \\
\text { Available }\end{array}$ \\
\hline R1 & DPW & $3 T$ & 50 \\
\hline R1 & DPW & $6 T$ & 80 \\
\hline R1 & DPW & $10 T$ & 30 \\
\hline R1 & UPDW & $3 T$ & 20 \\
\hline R1 & UPDW & $6 T$ & 30 \\
\hline
\end{tabular}




\begin{tabular}{|l|l|l|l|}
\hline R1 & UPDW & $10 T$ & 20 \\
\hline R2 & DPW & $3 T$ & 100 \\
\hline R2 & DPW & $6 T$ & 185 \\
\hline R2 & DPW & $10 T$ & 55 \\
\hline R2 & UPDW & $3 T$ & 20 \\
\hline R2 & UPDW & $6 T$ & 20 \\
\hline R2 & UPDW & $10 T$ & 10 \\
\hline R3 & DPW & $3 T$ & 110 \\
\hline R3 & DPW & $6 T$ & 250 \\
\hline R3 & DPW & $10 T$ & 150 \\
\hline R3 & UPDW & $3 T$ & 20 \\
\hline R3 & UPDW & $6 T$ & 30 \\
\hline R3 & UPDW & $10 T$ & 10 \\
\hline R1 & RW & $6 T$ & 30 \\
\hline R1 & RW & $10 T$ & 50 \\
\hline R2 & RW & $6 T$ & 45 \\
\hline R2 & RW & $10 T$ & 60 \\
\hline R3 & RW & $6 T$ & 65 \\
\hline R3 & RW & $10 T$ & 80 \\
\hline
\end{tabular}

Table S10: Assumed Breakdown of total water usage according to purpose for each consumer

\begin{tabular}{|c|c|c|}
\hline Consumer & DPW & UPDW \\
\hline HHC & $95 \%$ & $5 \%$ \\
\hline CC & $99 \%$ & $1 \%$ \\
\hline HC & $0 \%$ & $100 \%$ \\
\hline
\end{tabular}

Table S11: Comparison of Tanker Trips and extra tankers hired in normal and maintenance scenario

\begin{tabular}{|c|c|c|c|c|c|c|c|}
\hline $\begin{array}{c}\text { Tanker } \\
\text { Type }\end{array}$ & Product & $\begin{array}{c}\text { Tanker } \\
\text { Capacit } \\
\mathbf{y}(\mathbf{L})\end{array}$ & $\begin{array}{c}\text { Total } \\
\text { Tankers } \\
\text { Availability }\end{array}$ & $\begin{array}{c}\text { Extra } \\
\text { Tankers } \\
\text { Hired } \\
\text { (Normal } \\
\text { Scenario) }\end{array}$ & $\begin{array}{c}\text { No. of Trips } \\
\text { (Normal } \\
\text { Scenario) }\end{array}$ & $\begin{array}{c}\text { Extra } \\
\text { Tankers } \\
\text { Hired } \\
\text { (Maintenance } \\
\text { Scenario) }\end{array}$ & $\begin{array}{c}\text { No. of Trips } \\
\text { (Maintenance } \\
\text { Scenario) }\end{array}$ \\
\hline 3T & DPW & 3000 & 260 & 7 & 8.34 & 0 & 6.99 \\
\hline 3T & UPDW & 3000 & 60 & 0 & 4.96 & 0 & 2.17 \\
\hline 6T & DPW & 6000 & 515 & 163 & 10.10 & 274 & 8.99 \\
\hline 6T & UPDW & 6000 & 80 & 0 & 5.66 & 40 & 3.95 \\
\hline
\end{tabular}




\begin{tabular}{|c|c|c|c|c|c|c|c|}
\hline $10 \mathrm{~T}$ & DPW & 10,000 & 235 & 0 & 10.7 & 0 & 10.6 \\
\hline $10 \mathrm{~T}$ & UPDW & 10,000 & 40 & 0 & 8.75 & 0 & 9.7 \\
\hline $10 \mathrm{~T}$ & RW & 10,000 & 190 & 0 & 9.52 & 0 & 6.75 \\
\hline
\end{tabular}

Specimen of expanding constraint equations in the formulation:

$$
\begin{gathered}
x Q_{s, i, p, t}=x Q_{s, i, p, t-1}+y P S l_{s, p, t} * S T p t_{s}-\sum_{c}^{c} x \operatorname{DeCon}_{s, c, p, t} \\
\forall s\left(: S T y_{s}={ }^{\prime} T F^{\prime}\right), i\left(: i={ }^{\prime} T W I^{\prime}\right), \\
p\left(: p \in P^{F} \text { and } S I P_{s, i, p}=1\right), t(: t=2 . . N T)
\end{gathered}
$$

This equation can be expanded for $s=\mathrm{TF} 2, \mathrm{TF} 1, i=\mathrm{TWI}, p=\mathrm{UPDW}, \mathrm{DPW}, t=76$ and $S T p t_{\text {TF2 }}=80 \mathrm{KL} / \mathrm{hr}$ and $S T p t_{\text {TF2 }}=50 \mathrm{KL} / \mathrm{hr}$ as follows:

TF Mass Balance (TF2, TWI, UPDW, 76):

$x Q(T F 2, T W I, U P D W, 76)-x Q(T F 2, T W 1, U P D W, 75)-80 * y P S I(T F 2, U P D W, 76)+$ $x$ DeCon (TF2, HHC1,UPDW,76) $+x$ DeCon $(T F 2, H C 1, U P D W, 76)+x D e C o n(T F 2, C C 1, U P D W, 76)+$ $x$ DeCon $(T F 2, H H C 2, U P D W, 76)+x D e C o n(T F 2, C C 2, U P D W, 76)+x D e C o n(T F 2, H H C 3, U P D W, 76)+$ $x$ DeCon $(T F 2, H C 2, U P D W, 76)+x D e C o n(T F 2, C C 3, U P D W, 76)+x$ DeCon $(T F 2, H C 3, U P D W, 76)=0$

TF Mass Balance (TF2, TWI, DPW, 76):

$x Q(T F 2, T W I, D P W, 76)-x Q(T F 2, T W I, D P W, 75)-80 * y P S I(T F 2, D P W, 76)+x D e C o n(T F 2, H H C 1, D P W, 76)+$ $x$ DeCon $(\mathrm{TF} 2, \mathrm{HC1}, \mathrm{DPW}, 76)+x \mathrm{DeCon}(\mathrm{TF} 2, \mathrm{CC} 1, \mathrm{DPW}, 76)+x \mathrm{DeCon}(\mathrm{TF} 2, \mathrm{HHC} 2, \mathrm{DPW}, 76)+x \mathrm{DeCon}$ $(\mathrm{TF} 2, \mathrm{CC} 2, \mathrm{DPW}, 76)+x \mathrm{DeCon}(\mathrm{TF} 2, \mathrm{HHC} 3, \mathrm{DPW}, 76)+x \mathrm{DeCon}(\mathrm{TF} 2, \mathrm{HC2}, \mathrm{DPW}, 76)+x \mathrm{DeCon}$ $(\mathrm{TF} 2, \mathrm{CC} 3, \mathrm{DPW}, 76)+x \mathrm{DeCon}(\mathrm{TF} 2, \mathrm{HC} 3, \mathrm{DPW}, 76)=0$

\section{TF Mass Balance (TF1, TWI, UPDW, 76):}

$x Q(T F 1, T W I, U P D W, 76)-x Q(T F 1, T W I, U P D W, 75)-50 * y P S I(T F 1, U P D W, 76)+$ $x \operatorname{DeCon}(\mathrm{TF} 1, \mathrm{HHC1}, \mathrm{UPDW}, 76)+x \operatorname{DeCon}(\mathrm{TF} 1, \mathrm{HC1}, \mathrm{UPDW}, 76)+x \operatorname{DeCon}(\mathrm{TF1}, \mathrm{CC} 1, \mathrm{UPDW}, 76)+$ $x$ DeCon $(\mathrm{TF} 1, \mathrm{HHC2}, \mathrm{UPDW}, 76)+x \operatorname{DeCon}(\mathrm{TF} 1, \mathrm{CC} 2, \mathrm{UPDW}, 76)+x$ DeCon $(\mathrm{TF} 1, \mathrm{HHC} 3, \mathrm{UPDW}, 76)+$ $x$ DeCon $(\mathrm{TF} 1, \mathrm{HC2}, \mathrm{UPDW}, 76)+x$ DeCon $(\mathrm{TF1}, \mathrm{CC} 3, \mathrm{UPDW}, 76)+x \operatorname{DeCon}(\mathrm{TF1}, \mathrm{HC} 3, \mathrm{UPDW}, 76)=0$

TF Mass Balance (TF1, TWI, DPW, 76):

$x Q(T F 1, T W I, D P W, 76)-x Q(T F 1, T W I, D P W, 75)-50 * y P S I(T F 1, D P W, 76)+x D e C o n(T F 1, H H C 1, D P W, 76)+$ $x$ DeCon $(\mathrm{TF} 1, \mathrm{HC1}, \mathrm{DPW}, 76)+x \mathrm{DeCon}(\mathrm{TF} 1, \mathrm{CC} 1, \mathrm{DPW}, 76)+x \mathrm{DeCon}(\mathrm{TF} 1, \mathrm{HHC} 2, \mathrm{DPW}, 76)+x \mathrm{DeCon}$ $(\mathrm{TF} 1, \mathrm{CC} 2, \mathrm{DPW}, 76)+x \mathrm{DeCon}(\mathrm{TF} 1, \mathrm{HHC} 3, \mathrm{DPW}, 76)+x \mathrm{DeCon}(\mathrm{TF} 1, \mathrm{HC} 2, \mathrm{DPW}, 76)+x \mathrm{DeCon}$ $(\mathrm{TF} 1, \mathrm{CC} 3, \mathrm{DPW}, 76)+x \mathrm{DeCon}(\mathrm{TF} 1, \mathrm{HC} 3, \mathrm{DPW}, 76)=0$ 


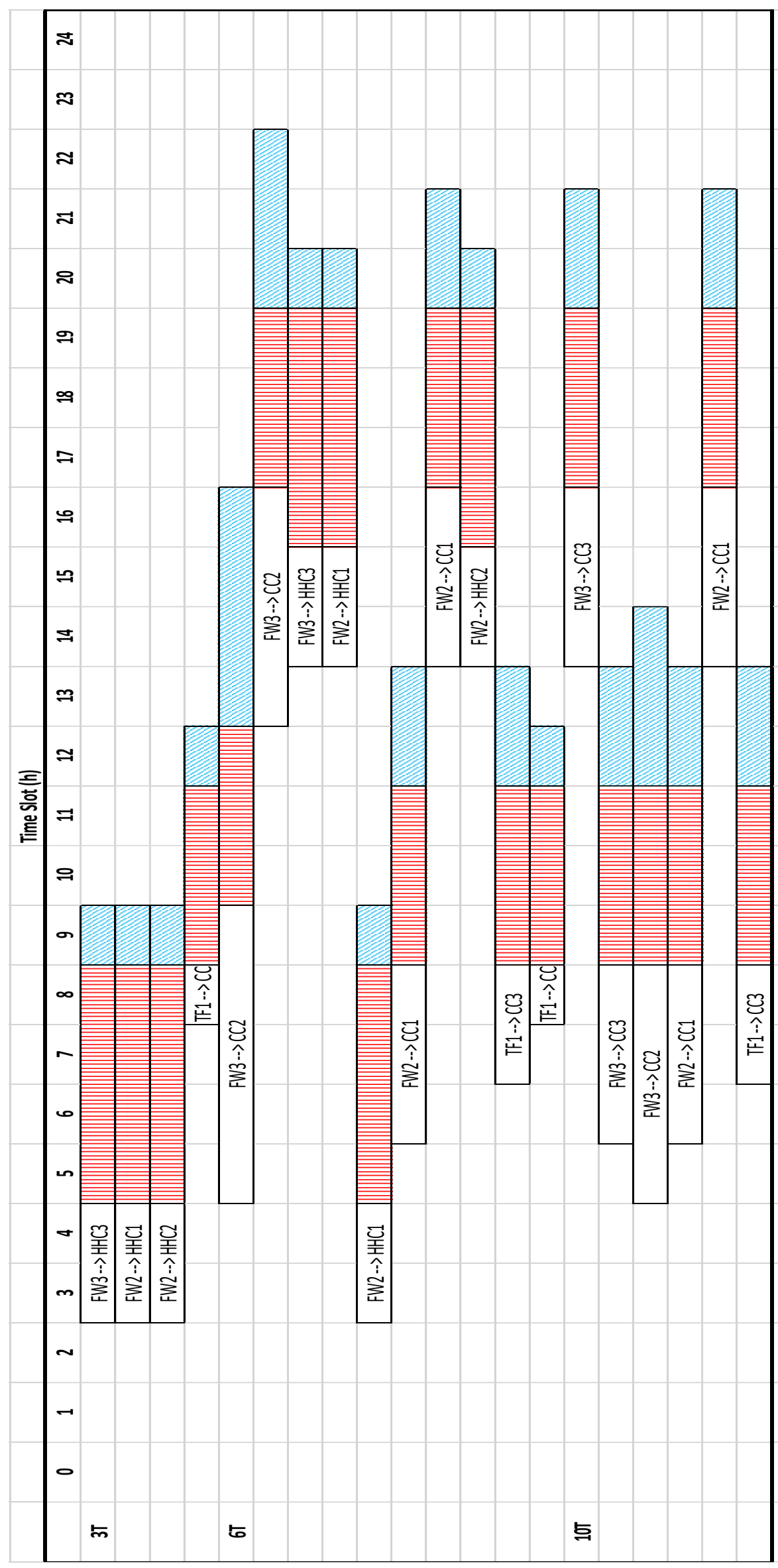

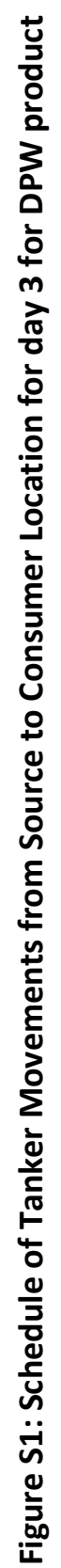

